\title{
Publisher's Note: Color screening in flux tubes and in the color Coulomb potential from QCD field correlators [Phys. Rev. D 96, 076019 (2017)]
}

M. S. Lukashov and Yu. A. Simonov

Q (Received 2 November 2018; published 19 November 2018)

DOI: 10.1103/PhysRevD.98.099903

This paper was published online on 30 October 2017 with a tagging error in an author affiliation. Yu. A. Simonov's affiliation should read as "1 Alikhanov Institute for Theoretical and Experimental Physics, B. Cheremushkinskya 25, 117218 Moscow, Russia." The author affiliation has been corrected as of 7 November 2018. The author affiliation is incorrect in the printed version of the journal. 